Supporting information for

\title{
Characterization of Individual Magnetic Nanoparticles in Solution by Double Nanohole Optical Tweezers
}

Haitian Xü Steven Jones ${ }^{\dagger}$, Byoung-Chul Choi $i^{\dagger}$ and Reuven Gordon ${ }^{t, *}$

${ }^{\dagger}$ Department of Physics and Astronomy, University of Victoria, Victoria V8P 5C2, Canada

${ }^{\ddagger}$ Department of Electrical and Computer Engineering, University of Victoria, Victoria V8P 5C2, Canada

*E-mail:rgordon@uvic.ca. 


\section{Effect of trapped nanoparticle on local field}

The effect of the nanoparticle (NP) on the local field of the double-nanohole (DNH) was investigated using a commercial finite-difference time-domain package ${ }^{1}$ with imported SEM images of the DNH and a mesh size of $1 \mathrm{~nm}$ in the vicinity of the DNH cusp. The local $|E|$ field profile and transmission spectra are shown below in Figures S1, S2.

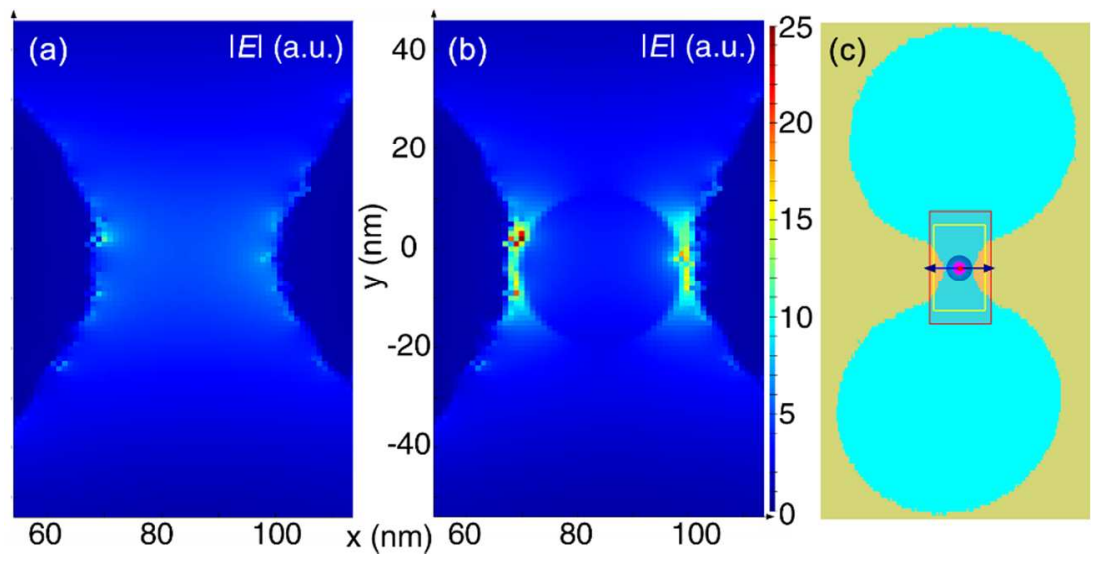

Figure. S1 Local $|E|$ profile in the cusp of DNH (a) without and (b) with $30 \mathrm{~nm}$ magnetite NP at the experimental laser frequency of $855 \mathrm{~nm}$. (c): Simulation cross-section, showing the incident $E$-field orientation and the DNH imported from SEM image. A mesh override region (1 nm mesh size) in the vicinity of the cusp is highlighted. The presence of the NP dramatically y enhances the local $E$-field in the cusp. 


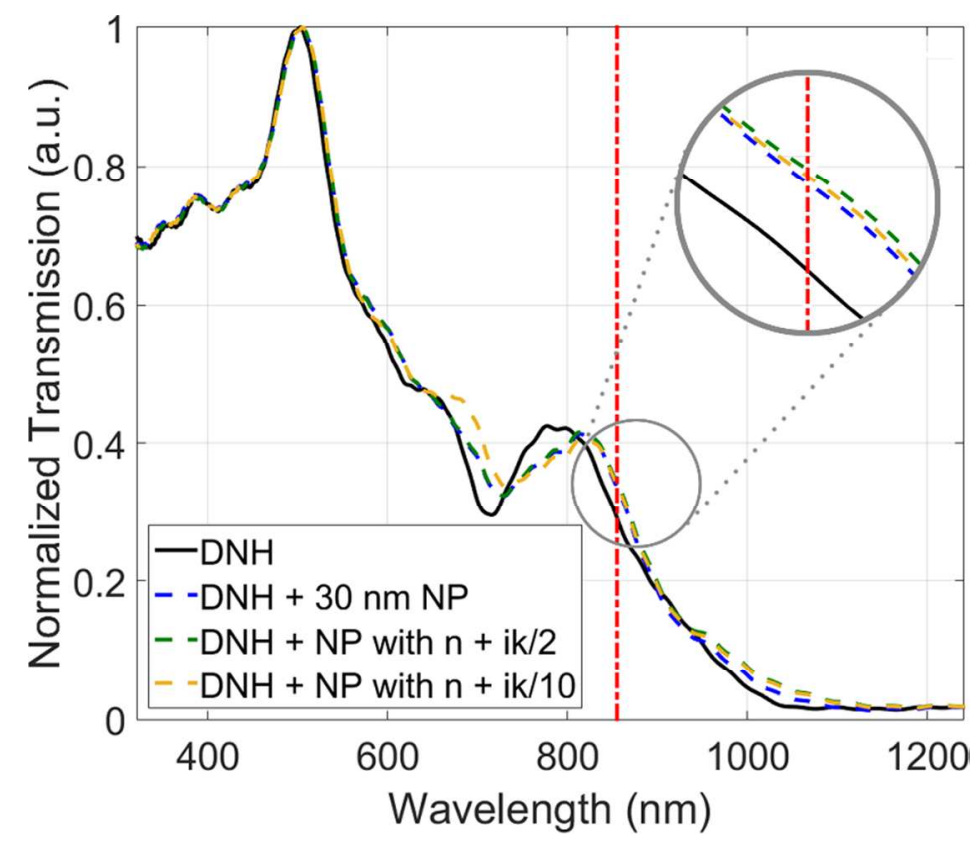

Figure. S2 Normalized transmission spectra of the DNH (black), DNH + trapped NP (blue), DNH + trapped NP with reduced extinction coefficients $k / 2$ and $k / 10$ (green, yellow). The experimental laser wavelength ( $855 \mathrm{~nm}$, dotted red vertical line) is red-detuned with respect to the $\mathrm{DNH}$ plasmon resonance. ${ }^{2}$ The presence of the NP red-shifts the resonance towards the laser wavelength, giving rise to increased transmission. The simulations with reduced $k$ show negligible effects on the increase in transmission at $855 \mathrm{~nm}$. 


\section{Calculating refractive index $n$}

Magnetite $(n, k)$ vs. wavelength

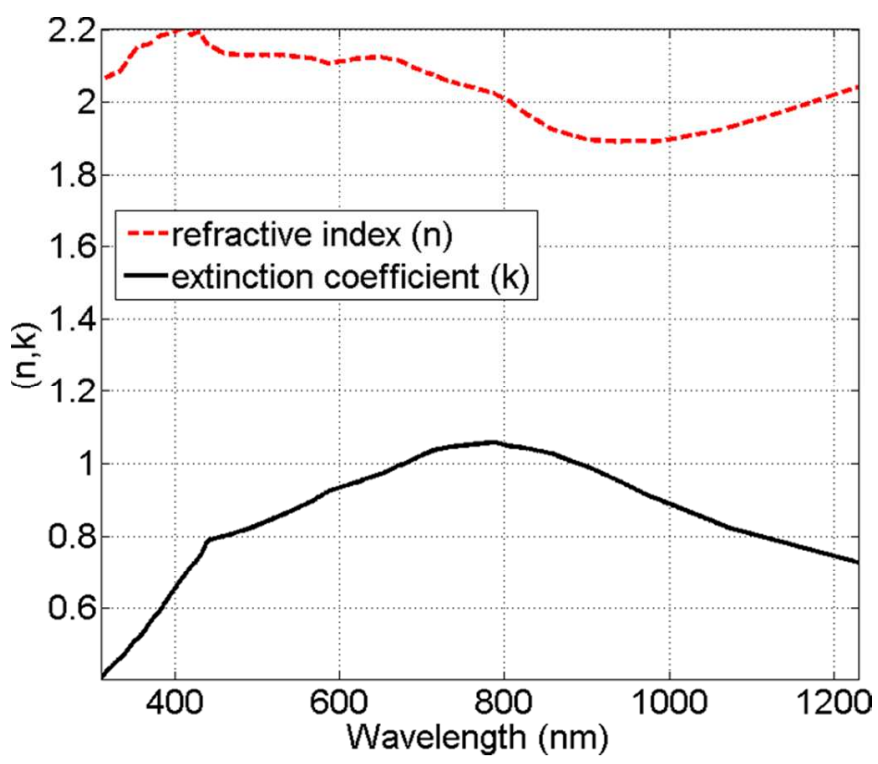

Figure. S3 $(n, k)$ vs. wavelength for magnetite, calculated from data presented in Schlegel et al., ${ }^{3}$ At the experimental laser wavelength of $855 \mathrm{~nm}, n \approx 1.93, k \approx 1.02$.

Refer to Figure 3 in the main article: transmission step size is determined by dielectric loading and absorption decrease when the NP becomes trapped. Its relation with the Clausius-Mossotti (CM) factor $K$ is complicated by the imaginary part of $K$. Our simulation results with varying extinction coefficient $k$ (Figure S2) show that the increase in transmission is determined by the refractive index $n$, with $k$ having a negligible impact at our experimental wavelength of $855 \mathrm{~nm}$. We can therefore assume the step size scales linearly with the CM factor of refractive indices.

For polystyrene (PS):

$$
K_{P S}=\frac{1.57^{2}-1.33^{2}}{1.57^{2}+2 \times 1.33^{2}}=0.116
$$

For magnetite (mag):

$$
\frac{K_{\mathrm{mag}, \mathrm{n} \text { only }}}{K_{\mathrm{PS}, \mathrm{n} \text { only }}}=\frac{\left(\frac{n_{\mathrm{mag}}^{2}-1.33^{2}}{n_{\mathrm{mag}}^{2}+2 \times 1.33^{2}}\right)}{0.116}=\frac{11.2}{5.1} \rightarrow n_{\mathrm{mag}} \approx 1.89 .(\mathrm{cf} .1 .93)
$$




\section{Calculating trap stiffness $\kappa$}

APD signal fluctuations are the result of Brownian motion of the trapped NP under the influences of restoring optical force $-\kappa x$ and viscous drag. Taking $r=15 \mathrm{~nm}, h=20 \mathrm{~nm}$, and $\eta_{\text {water }}=8.9 \times 10^{-4} \mathrm{Ns} / \mathrm{m}^{2}$, equation (5) gives the Stokes drag coefficient $\gamma$ :

$$
\gamma=\frac{6 \pi \times 8.9 \times 10^{-4} \times 15 \times 10^{-9}}{\left[1-\frac{9}{16}\left(\frac{15}{20}\right)+\frac{1}{8}\left(\frac{15}{20}\right)^{3}-\frac{45}{256}\left(\frac{15}{20}\right)^{4}-\frac{1}{16}\left(\frac{15}{20}\right)^{5}\right]}=4.49 \times 10^{-10} \mathrm{Ns} / \mathrm{m}
$$

The characteristic time of autocorrelation decay $\tau_{\mathrm{a}}$ averaged over multiple trapping events was measured to be $0.44 \mathrm{~ms}$. The trap stiffness can then be calculated from equation (4):

$$
\tau_{a}=\frac{\gamma}{\kappa} \rightarrow \kappa=\frac{\gamma}{\tau_{a}}=\frac{4.49 \times 10^{-10}}{0.44 \times 10^{-3}}=1.02 \times 10^{-6} \mathrm{Nm}^{-1}=1.02 \mathrm{fNnm}^{-1}
$$

Similarly, for the reference polystyrene NP, we obtain $\tau_{\mathrm{a}}=1.19 \mathrm{~ms}$ and $\kappa=0.38 \mathrm{fNnm}^{-1}$.

\section{Calculating extinction coefficient $k$}

The restoring gradient force due to dielectrophoresis, $\mathrm{F}_{\mathrm{DEP}}$ acting on a trapped nanoparticle with radius $r$ in a medium $\varepsilon_{\mathrm{m}}$ is given by: ${ }^{4}$

$$
F_{D E P}=2 \pi r^{3} \varepsilon_{m} R e[K] \nabla E^{2},
$$

where $\operatorname{Re}[K]$ is the real part of the complex CM factor. Since magnetite and the reference polystyrene NPs have (nominally) the same size, are immersed in the same medium at the same temperature under the same trapping laser intensity, their restoring forces, hence stiffnesses, scale linearly with $\operatorname{Re}[K]$, where $\operatorname{Re}\left[K_{\mathrm{PS}}\right]=K_{\mathrm{PS}}=0.116$ from Section 2 .

For magnetite (mag) in water at $855 \mathrm{~nm}$ :

$$
K=\frac{\tilde{\varepsilon}_{p}-\varepsilon_{m}}{\tilde{\varepsilon}_{p}+2 \varepsilon_{m}}=, \frac{\left(n_{\mathrm{mag}}+\mathrm{i} k_{\mathrm{mag}}\right)^{2}-1.33^{2}}{\left(n_{\mathrm{mag}}+\mathrm{i} k_{\mathrm{mag}}\right)^{2}+2 \times 1.33^{2}}, n_{\mathrm{mag}}=1.89 \text { from Section } 2 .
$$

We can now use $\kappa$ (stiffness) ratios between mag and PS to find $k_{\text {mag: }}$ : 


$$
\frac{\kappa_{\mathrm{mag}}}{k_{\mathrm{PS}}}=\frac{1.021}{0.377}=\frac{\operatorname{Re}\left[\frac{\left(1.89+\mathrm{i} k_{\mathrm{mag}}\right)^{2}-1.33^{2}}{\left(1.89+\mathrm{i} k_{\mathrm{mag}}\right)^{2}+2 \times 1.33^{2}}\right]}{0.116} \rightarrow k_{\mathrm{mag}} \approx 0.73 \text { (cf. } 1.02 \text { reference data) }
$$

\section{Size characterization}

\section{Single trapping event}

Refer to Figure 6(a) and equation (10) in the main article: to obtain a more accurate measurement of diameter $d$, the applied field was cycled through several values before the trapped nanoparticle would escape, and $d$ was calculated for each applied field value. Uncertainty in $d$ was estimated as $\sigma / \sqrt{ } N$, where $\sigma$ is the standard deviation in the average and $N$ is the number of field values used. Figure $\mathrm{S} 1$ shows example measurements for a trapping event.

\begin{tabular}{|c|c|}
\hline Field (Oe) & Diameter (nm) \\
\hline 107.2 & 31.02 \\
\hline 142.0 & 31.79 \\
\hline 175.2 & 30.81 \\
\hline 240.4 & 29.57 \\
\hline 272.4 & 30.81 \\
\hline 303.6 & 30.72 \\
\hline 334.0 & 30.47 \\
\hline Average & $\mathbf{3 0 . 7 4}$ \\
\hline Uncertainty & $\mathbf{0 . 2 5}$ \\
\hline
\end{tabular}


Table. S1 Single trapping event: calculated diameters of the trapped magnetite nanoparticle under different applied fields.

\section{Multiple trapping events}

\begin{tabular}{|c|c|}
\hline Trapping Event \# & Calculated Nanoparticle Diameter (nm) \\
\hline 1 & $29.63 \pm 0.57$ \\
\hline 2 & $45.43 \pm 0.67$ \\
\hline 3 & $29.53 \pm 0.22$ \\
\hline 4 & $43.09 \pm 0.95$ \\
\hline 5 & $29.12 \pm 0.27$ \\
\hline 6 & $30.21 \pm 0.27$ \\
\hline 7 & $27.15 \pm 0.19$ \\
\hline 8 & $32.91 \pm 0.47$ \\
\hline 9 & $30.74 \pm 0.25$ \\
\hline 10 & $31.37 \pm 0.34$ \\
\hline 11 & $31.97 \pm 0.28$ \\
\hline 12 & $30.71 \pm 0.38$ \\
\hline 13 & $32.57 \pm 0.46$ \\
\hline 14 & $33.84 \pm 0.24$ \\
\hline
\end{tabular}

Table. S2 Multiple trapping events: calculated diameters for 14 separate trapping events. Shaded rows are possibly due to trapped aggregate particles - e.g., dimer/trimers, much larger than the average 
nanoparticle. These are occasionally observed in the SEM (Figure S3). They are omitted in the main article.

\section{Magnetite aggregate nanoparticle SEM images}

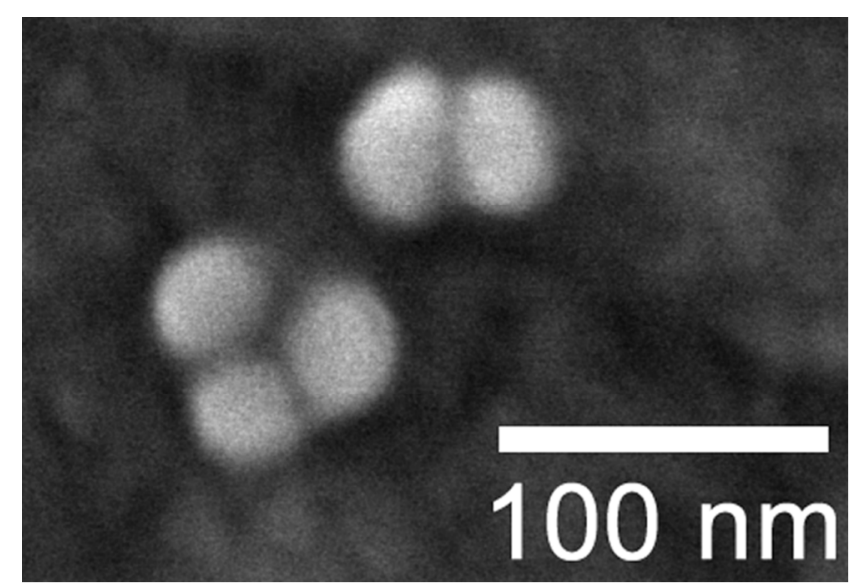

Figure. S4 SEM image of dimer and trimer $\mathrm{Fe}_{3} \mathrm{O}_{4}$ aggregate nanoparticle.

\section{References}

(1) Lumerical Solutions, Inc. http://www.lumerical.com/tcad-products/fdtd/.

(2) Mestres, P.; Berthelot, J.; Quidant, R. arXiv:1511.05310 2015.

(3) Schlegel, A.; Alvarado, S. F.; Wachter, P. J. Phys. C Solid State Phys. 1979, 12, 1157.

(4) Castellarnau, M.; Errachid, A.; Madrid, C.; Juárez, A.; Samitier, J. Biophys. J. 2006, 91, $3937-$ 3945 . 